\title{
Consensus Control of Linear Multi-Agent Systems under Directed Dynamic Topology
}

\author{
Jiahu Qin ${ }^{1}$ and Changbin $\mathrm{Yu}^{2}$
}

\begin{abstract}
This paper aims to extend the nonnegative matrix theory, which is widely employed for multiple integrator agents, to deal with the consensus control of generic linear multiagent systems (MASs) under directed dynamic topology. It is finally shown that the exponential consensus can be reached under very relaxed conditions, i.e., the directed interaction topology is only required to be repeatedly jointly rooted and the exponentially unstable mode of each individual system is weak enough. Moreover, a least convergence rate and a bound for the unstable mode of the individual agent system, both of which are independent of the switching mode, can be explicitly specified.
\end{abstract}

\section{INTRODUCTION}

One of the key problems in multi-agent consensus is to find the weakest possible conditions that need to be imposed on the interaction topology to guarantee the desired consensus behavior [2], [4], [6]-[8], [13], [15], [16], [25]. It is well known that to reach the consensus, having a rooted graph (also called having a directed spanning tree) is the weakest condition [16] on the fixed directed interaction topology, while having a repeatedly jointly rooted interaction topology is the weakest possible condition for the dynamic case [6], [10], [16]. Nonnegative matrix theory, in particular the product properties of infinite row-stochastic matrices [23], is demonstrated being the most effective and popular analysis tool in dealing with the convergence analysis for a group of linearly coupled agents under dynamic topology [6], [15]-[17].

Very recently, much attention is switched to the coordination control of generic linear MASs which takes more general form and thus can be used to model more complicated scenarios in real applications. In this framework, each agent, referred to as generic linear agent for clarity, is modeled by the following dynamics:

$$
\dot{x}_{i}=A x_{i}+B u_{i}, \quad i=1,2, \ldots, N,
$$

where $x_{i} \in \mathbb{R}^{n}$ is the $i$ th agent's state, $B \in \mathbb{R}^{n \times m}, u_{i} \in \mathbb{R}^{m}$ is the control input for agents $i$. Different from integrator agents, the collective behavior of generic linear MASs is determined not only by the dynamical rules governing the isolated agents, modeled by e.g., $\dot{x}_{i}(t)=A x_{i}(t)$ in this paper, but also by the interactions between the neighboring

\footnotetext{
${ }^{1}$ J. Qin is with the Australian National University, Canberra, Australia jiahu.qin canu.edu.au

${ }^{2} \mathrm{C}$. Yu is with the Australian National University and NICTA Ltd, Canberra, A.C.T., Australia, and also with Shandong Computer Science Center, Jinan, China. The work of C. Yu was supported by the Australian Research Council through DP-130103610 and a Queen Elizabeth II Fellowship under DP-110100538, and the Overseas Expert Program of Shandong Province brad.yu ¿anu.edu.au
}

agents. This makes the coordination control for generic linear agents much more challenging than that for the integrator agents. Some efforts made towards tackling the consensus problem of system (1) by using static distributed feedback controller can be found in, e.g., [11], [14], [18], [19], [22].

Consensus/synchronization under fixed interaction topology is investigated in [18], [24] in discrete-time setting and in [14], [19] in continuous-time setting. Similar to that required for integrator agents, having a rooted graph is shown to be the weakest condition that should be imposed on the interaction topology to guarantee the consensus. Considering, as mentioned earlier, the analysis techniques for integrator agents cannot be extended straightforwardly to the generic linear agents, [22] and [11] investigate the consensus problem for generic linear MASs under undirected dynamic topology using developed Lypaunov stability theory which, however, relies heavily on the symmetric property of the associated Laplacian matrix, and thus confining its applicability to the case with directed interaction topology. Specifically, interaction topology is required to be frequently connected in [22], and this restrictive condition is later relaxed to a jointly connected one in [11], which is only valid in a leader-following framework. One can easily observe from [11] that the proof technique does not work for the case without a leader.

Noting on one hand that real-world information exchanges between agents may be unidirectional and thus results in the directed interaction topology, and on the other hand that the leader-following scenario is a special case of the leaderless consensus to be considered, this paper aims to investigate the consensus control of generic linear MASs under a very general setting, i.e. leaderless consensus control under directed dynamic topology. The contribution of our work comparing to the existing ones comprising mainly the following two points:

(a) Totally different convergence analysis for generic linear MASs from the existing ones is used in our work. We attempt to bridge the gaps between the consensus analysis for integrator agents, see e.g. [6], [15], [16], and that for generic linear agents under dynamic topology. Toward this end, nonnegative matrix theory, in particular the product properties of infinite row-stochastic matrices, is developed combined with some other matrix analysis techniques to deal with consensus of generic linear MASs. Instead of performing only the asymptotical consensus analysis, we consider in this paper the exponential consensus and further specify a least convergence rate under dynamic topology. This generalizes the existing convergence analysis in [6], 
[15], [16] to very general settings.

(b) Consensus control is investigated upon a very general setting. The weighting factors are allowed to change dynamically to model more practical dynamics and the interaction topology switches among an infinite set of weighted directed graphs as opposed to the finite undirected ones in [22] and [11]. Moreover, interaction topology is only required to be repeatedly jointly rooted. This extends the assumption on the interaction topology concerning dynamic case in [11], [22] to a more general setting although at the cost of the full-state coupling between agents, it comes out with a more relaxed assumption on $A$ than that in [11], [22]. More specifically, $A$ is allowed to have exponentially unstable mode and a upper bound for such unstable mode as well as the convergence rate can be specified as well.

\section{PReliminaries}

\section{A. Graph and Matrix Notation}

The following notations will be used throughout the paper. Let $|\cdot|$ denote the 2-norm of a vector, and $\|\cdot\|_{F}$ denote the Frobenius norm of a matrix. Identity matrix in $\mathbb{R}^{p \times p}$ is denoted by $I_{p}$ and zero matrix in $\mathbb{R}^{p \times q}$ by $0_{p \times q}$. Let $\mathbf{1}_{N}\left(0_{N}\right)$ be the column vector with all entries equal to $1(0), \operatorname{Re}(\lambda)$ be the real part of complex number $\lambda$. When the subscripts $m$ and $n$ are dropped, the dimensions of these vectors and matrices are assumed to be compatible with the context. Denote by $M>0(M<0)$ that $M$ is symmetric positive (negative) definite. Denote by $\operatorname{diag}\left\{A_{1}, A_{2}, \ldots, A_{n}\right\}$ the block diagonal matrix with its $i$ th main diagonal matrix being a square matrix $A_{i}, i=1, \ldots, n$. A matrix $M$ is said to be nonnegative if all its entries are nonnegative. Let $\otimes$ be the Kronecker product and the Kronceker product has the following property which will be used throughout the analysis.

Lemma 1: For any two matrices $A$ and $B$, we have $\| A \otimes$ $B\left\|_{F}=\right\| A\left\|_{F}\right\| B \|_{F}$.

The readers are referred to [9] for more properties concerning the Kronecker product.

A nonnegative matrix $M$ is said to be row stochastic if all its row sums are 1 . Let $S_{d}$ denote the set of $N$ by $N$ row-stochastic matrices with positive diagonal elements, and $S_{d}(v)$ denote the matrices in $S_{d}$ with each nonzero element being larger than or equal to $v$. A row-stochastic matrix $M$ is called indecomposable and aperiodic (SIA) [23] if there exists a column vector $v$ such that $\lim _{k \rightarrow \infty} M^{k}=1 v^{\mathrm{T}}$. Let $\prod_{i=1}^{k} M_{i}=M_{k} M_{k-1} \cdots M_{1}$ denote the left product of the matrices $M_{k}, M_{k-1}, \cdots, M_{1}$. Two nonnegative matrices $M$ and $N$ are said to be of the same type, denoted by $M \sim N$, if they have zero elements and positive elements in the same places.

Given any $N \times N$ stochastic matrix $S=\left[s_{i j}\right]$ [23], $\lambda(s)$ is defined by

$$
\lambda(S)=1-\min _{i_{1}, i_{2}} \sum_{j=1}^{N} \min \left\{s_{i_{1} j}, s_{i_{2} j}\right\},
$$

and $\delta(S)$ by

$$
\delta(S)=\max _{j} \max _{i_{1}, i_{2}}\left|s_{i_{1} j}-s_{i_{2} j}\right| .
$$

$\delta(S)$ measures, in a certain sense, how different the rows of $S$ are, and clearly $\delta(S)=0$ if and only if the rows of $S$ are identical. A matrix $S$ is called scrambling if $\lambda(S)<1$. $\lambda(S)=0$ if and only if $\delta(S)=0$.

The following is a result specifying the relation between functions $\delta(\cdot)$ and $\lambda(\cdot)$.

Lemma 2: ( [23], Lemma 2) For any row-stochastic matrices $P_{1}, P_{2}, \ldots, P_{k}$,

$$
\delta\left(P_{1} P_{2} \cdots P_{k}\right) \leq \prod_{i=1}^{k} \lambda\left(P_{i}\right) .
$$

Let $\mathbb{A}=\left\{A_{1}, \ldots, A_{k}\right\}$ ( $\mathbb{A}$ can be an infinite set) be a set of square matrices with the same order. By a word (in the $A^{\prime} \mathrm{s}, A \in \mathbb{A}$ ) of length $m$ we mean the product of $m$ $A^{\prime}$ s (repetitions permitted) [23]. Then, we have the following result

Lemma 3: ( [21]) Let $S$ be a set of $N$ by $N$ SIA matrices with positive diagonal elements, then any word in the $s$ 's ( $s \in S$ ) of length $N-1$ or larger is scrambling.

Let digraph $G=(\mathcal{V}, \varepsilon)$ consisting of a node set $\mathcal{V}=$ $\{1, \ldots, N\}$ and an edge set $\varepsilon \subset \mathcal{V} \times \mathcal{V}$ represent the interaction topology among a group of $N$ generic linear agents, and $\mathcal{A}=\left[a_{i j}\right] \in \mathbb{R}^{N \times N}$ be the associated adjacency matrix in which $a_{i j}>0 \Leftrightarrow(j, i) \in \varepsilon$. Moreover, we assume $a_{i i}=0, i=1, \ldots, N$. Denote by $L=\left[\ell_{i j}\right]$ the Laplacian matrix associated with $G$, where $\ell_{i j}=-a_{i j}, i \neq j$, and $l_{i i}=\sum_{k=1, k \neq i}^{N} a_{i k}$ [5]. $G$ is called a rooted graph or a graph has a directed spanning tree if there exists at least one node, called the root node, having a directed path to all other nodes.

\section{B. Distributed Control Algorithm}

The distributed feedback control law for agent $i$ is considered as follows:

$$
u_{i}(t)=K \sum_{j \in N_{i}(t)} a_{i j}(t)\left(x_{j}(t)-x_{i}(t)\right), \quad i=1,2, \ldots, N,
$$

where $a_{i j}(t)>0$ if agent $i$ can receive the information of agent $j$ at time $t$ while $a_{i j}(t)=0$ otherwise, and $N_{i}(t)=$ $\left\{j \in \mathcal{V}: a_{i j}(t)>0\right\}$ is the set of neighbors of agent $i$ at time $t$. In particular, it is assumed that all the nonnegative and thus positive weighting factors $a_{i j}(t)$ 's are uniformly lower and upper bounded (i.e., $a_{i j}(t) \in[\underline{a}, \bar{a}]$, where $0<\underline{a}<\bar{a}$, if $a_{i j}(t) \neq 0$ ). $K \in \mathbb{R}^{m \times n}$ is a state feedback matrix to be designed.

We say that consensus is achieved by using distributed control law (2) if there exists a feedback matrix $K$ such that for any initial states $x_{i}(0)$,

$$
\lim _{t \rightarrow \infty}\left|x_{i}(t)-x_{j}(t)\right|=0, \quad i, j \in \mathcal{V} .
$$




\section{MAIN RESUlTS}

In this section, we aim to explore the product properties of infinite row-stochastic matrices together with some other matrix analysis techniques to prove that consensus can be achieved by using distributed control law (2) if the interaction topology is only required to be repeatedly jointly rooted.

Note that system (1) can be rewritten in the following compact form

$$
\dot{x}(t)=\left(I_{N} \otimes A-L(t) \otimes B K\right) x(t),
$$

where $L(t)$ is the Laplacian matrix of $G(t)$, the interaction topology at time $t$. In fact, $L(t)$ specifies exactly how the time-varying system (3) switches. To make the convergence analysis mathematically tractable, we assume that $L(t)$ is piecewise continuous. Specifically, let $t_{0}, t_{1}, \ldots$ be an infinite time sequence at which the interaction topology $G(t)$ or weighting factors $a_{i j}(t)$ switch, where it is assumed that the dwell time $t_{i}-t_{i-1} \geq \tau>0, i=1,2, \ldots$. Then on each interval $\left[t_{i}, t_{j}\right)$, the interaction topology is fixed and $a_{i j}(t)$ is continuous.

Lemma 4: (cf. Lemma 2.23, [16]) Let $N$ by $N$ matrix $C(t)=\left[c_{i j}(t)\right]$ be piecewise continuous, where $c_{i j}>0$, $\forall i \neq j$, and $\sum_{j=1}^{N} c_{i j}=0$. Then the corresponding transition matrix $\Phi_{C}\left(t, t_{0}\right)$ of system $\dot{\zeta}(t)=C(t) \zeta(t)$ is a rowstochastic matrix with positive diagonal elements for any $t \geq t_{0}$.

To state the main result, we need to recall a few definitions and some results.

Lemma 5: Let $a$ be the maximum real parts of the eigenvalues of $N \times N$ matrix $A$, then there exists a $\gamma>0$ such that

$$
\left\|e^{A t}\right\|_{F} \leq \gamma t^{N-1} e^{a t}, \quad \forall t>1
$$

Denote $\bar{G}$ as the set of all possible interaction topologies among the $N$ agents ${ }^{1}$. The union of a group of digraphs $\left\{G_{i_{1}}, \ldots, G_{i_{k}}\right\} \subset \bar{G}$ is a digraph with the same node set and the edge set given by the union of the edge sets of $G_{i_{j}}$, $j=1, \ldots, k$.

An infinite sequence of interaction topologies $G\left(t_{0}\right), G\left(t_{1}\right), G\left(t_{2}\right), \ldots$, with the same node set is called repeatedly jointly rooted if there exists an infinite sequence of contiguous, nonempty, uniformly bounded time intervals $\left[t_{i_{j}}, t_{i_{j+1}}\right),\left(t_{i_{j+1}}-t_{i_{j}}<T\right) j=1,2, \ldots$, starting at $t_{i_{1}}=t_{0}$, for which each finite set of digraphs $G\left(t_{i_{j}}\right), G\left(t_{i_{j}+1}\right), \ldots, G\left(t_{i_{j+1}-1}\right)$ is jointly rooted [1], [6], [16].

We proceed to recall some results for consensus of multiple integrator agents. Let $\Phi\left(t, t_{0}\right)$ denote the transition matrix corresponding to system

$$
\dot{\xi}(t)=-L(t) \xi(t) .
$$

According to Lemma 4 , each transition matrix $\Phi\left(t_{i}, t_{i+1}\right)$, $i=0,1, \ldots$, is a row-stochastic matrix with positive diagonal elements.

Considering the graph structure of the interaction topology, there are only finite number, say $n_{0}$, of different types of

\footnotetext{
${ }^{1} \bar{G}$ is infinite since the set consisting of the weighting factors is infinite.
}

interaction topologies. As also stated in the proof of Theorem 2.33 in [17], for each possible interaction topology, $L(t)$ has the same structure across each interval $\left[t_{i}, t_{i+1}\right)$, i.e. $L\left(t_{1}\right) \sim L\left(t_{2}\right), \forall t_{1}, t_{2} \in\left[t_{i}, t_{i+1}\right)$ and each transition matrix $\Phi\left(t_{i}, t_{i+1}\right)$ is of constant type over this interval for each possible interaction topology. This together with the fact that $\Phi\left(t_{i}, t_{i+1}\right)$ is a continuous function of $l_{i j}(t)$ (note that $\left.L(t)=\left[l_{i j}(t)\right]\right)$ for $t \in\left[t_{i}, t_{i+1}\right)$ and $\tau \leq t_{i+1}-t_{i} \leq$ $T$ implies that for each possible interaction topology, all the nonzero and hence positive elements of $\Phi\left(t_{i}, t_{i+1}\right)$ are uniformly lower bounded, say by $\mu_{i}$. Apparently, $0<\mu_{i}<1$ since each $\Phi\left(t_{i}, t_{i+1}\right)$ is a row-stochastic matrix with positive diagonal elements. Let $\mu=\min \left\{\mu_{1}, \ldots, \mu_{n_{0}}\right\}$, then $0<$ $\mu<1$ and it follows straightforwardly that $\Phi\left(t_{i}, t_{i+1}\right) \in$ $S_{d}(\mu)$.

With the above preparations, we are now in a position to present our main result.

Theorem 1: Let $(A, B)$ be a matrix pair in which $B$ is with full row rank. If the interaction topologies $G\left(t_{0}\right), G\left(t_{1}\right), G\left(t_{2}\right), \ldots$, are repeatedly jointly rooted and matrix $A$ satisfies

$a=\max \{|\operatorname{Re}(\lambda)|: \lambda \in \sigma(A)\}<\frac{-\ln \left(1-\mu^{\lfloor T / \tau\rfloor(N-1)}\right)}{(N-1) T}$,

then there exists a feedback matrix $K=B^{\mathrm{T}}\left(B B^{\mathrm{T}}\right)^{-1}$ such that multi-agent system (1) can reach consensus exponentially fast with the least rate of

$$
a+\frac{\ln \left(1-\mu^{\lfloor T / \tau\rfloor(N-1)}\right)}{(N-1) T}
$$

by using distributed feedback controller (2).

Proof: Substitute $B^{\mathrm{T}}\left(B B^{\mathrm{T}}\right)^{-1}$ for $K$ in (3) yields

$$
\dot{x}(t)=\left(I_{N} \otimes A-L(t) \otimes I_{n}\right) x(t) .
$$

Denote by $\Psi\left(t, t_{0}\right)$ the transition matrix corresponding to system (5), then $x(t)=\Psi\left(t, t_{0}\right) x\left(t_{0}\right)$. In what follows, we first prove that $\Psi\left(t, t_{0}\right)=\Phi\left(t, t_{0}\right) \otimes e^{A\left(t-t_{0}\right)}$.

Noticing on one hand

$$
\frac{d}{d t} \Psi\left(t, t_{0}\right)=\left(I_{N} \otimes A-L(t) \otimes I_{n}\right) \Psi\left(t, t_{0}\right),
$$

and $\left.\Psi\left(t, t_{0}\right)\right|_{t=t_{0}}=I_{n N}$; and on the other hand

$$
\left.\Phi\left(t, t_{0}\right) \otimes e^{A\left(t-t_{0}\right)}\right|_{t=t_{0}}=I_{N} \otimes I_{n}=I_{n N},
$$

and

$$
\begin{aligned}
& \frac{d}{d t}\left(\Phi\left(t, t_{0}\right) \otimes e^{A\left(t-t_{0}\right)}\right) \\
= & \frac{d}{d t}\left(\Phi\left(t, t_{0}\right)\right) \otimes e^{A\left(t-t_{0}\right)}+\Phi\left(t, t_{0}\right) \otimes \frac{d}{d t}\left(e^{A\left(t-t_{0}\right)}\right) \\
= & \left(-L(t) \Phi\left(t, t_{0}\right)\right) \otimes e^{A\left(t-t_{0}\right)}+\Phi\left(t, t_{0}\right) \otimes\left(A e^{A\left(t-t_{0}\right)}\right) \\
= & \left(I_{N} \otimes A-L(t) \otimes I_{n}\right)\left(\Phi\left(t, t_{0}\right) \otimes e^{A\left(t-t_{0}\right)}\right) .
\end{aligned}
$$

Therefore, if follows straightforwardly that

$$
\Psi\left(t, t_{0}\right)=\Phi\left(t, t_{0}\right) \otimes e^{A\left(t-t_{0}\right)} .
$$

Apparently, $\Psi\left(t, t_{0}\right)$ is not row stochastic. 
Let $\Phi^{i_{j}}$ denote the transition matrix corresponding to system (4) for the union $\left[t_{i_{j}}, t_{i_{j+1}}\right)$, i.e.

$$
\Phi^{i_{j}}=\prod_{k=0}^{i_{j+1}-i_{j}-1} \Phi\left(t_{i_{j}+k}, t_{i_{j}+k+1}\right) .
$$

The condition that interaction topologies across each interval $\left[t_{i_{j}}, t_{i_{j+1}}\right)$ is jointly rooted implies that each $\Phi^{i_{j}}$, $j=1,2, \ldots$, is a SIA matrix with positive diagonal elements [6], [16].

With the above preparations, now consider

$$
W_{m}=\prod_{j=(m-1)(N-1)+1}^{m(N-1)} \Phi^{i_{j}}, \quad m=1,2, \ldots
$$

Obviously, $W_{m}$ is a word in the $\Phi$ 's $\left(\Phi \in\left\{\Phi^{i_{j}} \mid j=\right.\right.$ $1,2, \ldots\}$ ) of length $N-1$, it thus follows from Lemma 3 that each $W_{m} m=1,2, \ldots$, is a scrambling matrix, i.e., $\lambda\left(W_{m}\right)<1$. On the other hand, note that there are at most $\lfloor T / \tau\rfloor$ subintervals in each interval $\left[t_{i_{j}}, t_{i_{j+1}}\right)$ and each $\Phi\left(t_{\ell}, t_{\ell+1}\right) \in S_{d}(\mu), \mu=0,1, \ldots$, thus $\Phi^{i_{j}} \in S_{d}\left(\mu^{\lfloor T / \tau\rfloor}\right)$ and therefore $W_{m} \in S_{d}\left(\mu^{\lfloor T / \tau\rfloor(N-1)}\right)$. This in turn together with $\lambda\left(W_{m}\right)<1$ gives $\lambda\left(W_{m}\right) \leq 1-\mu^{\lfloor T / \tau\rfloor(N-1)}<1$.

Let $\beta=1-\mu^{\lfloor T / \tau\rfloor(N-1)}$. For any $j$ satisfying $j \geq 2 N-1$,

$$
\begin{aligned}
& \Phi\left(t_{i_{j+1}}, t_{i_{1}}\right) \\
= & \Phi\left(t_{i_{j+1}}, t_{i_{(N-1) \times\left\lfloor\frac{j-1}{N-1}\right\rfloor+1}}\right) \Phi\left(t_{i_{(N-1) \times\left\lfloor\frac{j-1}{N-1}\right\rfloor+1}}, t_{i_{1}}\right) \\
= & \Phi\left(t_{i_{j+1}}, t_{i_{(N-1) \times\left\lfloor\frac{j-1}{N-1}\right\rfloor+1}}\right) \cdot\left(\prod_{m=1}^{\left\lfloor\frac{j-1}{N-1}\right\rfloor} W_{m}\right),
\end{aligned}
$$

where $\left\lfloor\frac{j-1}{N-1}\right\rfloor$ denotes the maximum integer not greater than $\frac{j-1}{N-1}$. It then follows from the fact that any finite number of products of $\Phi^{i_{j}}$ is row-stochastic together with Lemma 2 that

$$
\begin{aligned}
& \delta\left(\Phi\left(t_{i_{j+1}}, t_{i_{1}}\right)\right) \\
& \leq \lambda\left(\Phi\left(t_{i_{j+1}, t_{(N-1) \times\left\lfloor\frac{j-1}{N-1}\right\rfloor+1}}\right)\right) \cdot\left(\prod_{m=1}^{\left\lfloor\frac{j}{N-1}\right\rfloor} \lambda\left(W_{m}\right)\right) \\
& \leq \prod_{m=1}^{\left\lfloor\frac{j}{N-1}\right\rfloor} \lambda\left(W_{m}\right) \leq \beta^{\left\lfloor\frac{j-1}{N-1}\right\rfloor} \leq \beta^{\frac{j-1}{N-1}-1} .
\end{aligned}
$$

This in turn implies that

$$
\lim _{j \rightarrow \infty} \delta\left(\Phi\left(t_{i_{j+1}}, t_{i_{1}}\right)\right)=0,
$$

i.e., the rows of $\Phi\left(t_{i_{j+1}}, t_{i_{1}}\right)$ approaches each other as $j \rightarrow$ $\infty$. This is equivalent to saying that $\Phi\left(t_{i_{j+1}}, t_{i_{1}}\right)$ converges to a rank one matrix of the form $1 v^{\mathrm{T}}$ [3], or in other words

$$
\left\|\Phi\left(t_{i_{j+1}}, t_{i_{1}}\right)-\mathbf{1} v^{\mathrm{T}}\right\|_{F}=\mathbf{O}\left(\delta\left(\Phi\left(t_{i_{j+1}}, t_{i_{1}}\right)\right)\right),
$$

where $\mathbf{O}(\cdot)$ stands for that $\left\|\Phi\left(t_{i_{j+1}}, t_{i_{1}}\right)-\mathbf{1} v^{\mathrm{T}}\right\|_{F}$ and $\delta\left(\Phi\left(t_{i_{j+1}}, t_{i_{1}}\right)\right)$ are equivalent infinitesimal.
Now we proceed to prove

$$
\begin{aligned}
& \lim _{j \rightarrow \infty}\left\|\left[\Phi\left(t_{i_{j+1}}, t_{i_{1}}\right)-\mathbf{1} v^{\mathrm{T}}\right] \otimes e^{A\left(t_{i_{j+1}}-t_{i_{1}}\right)}\right\|_{F} \\
= & \lim _{j \rightarrow \infty}\left\|\Phi\left(t_{i_{j+1}}, t_{i_{1}}\right)-\mathbf{1} v^{\mathrm{T}}\right\|_{F} \cdot\left\|e^{A\left(t_{i_{j+1}}-t_{i_{1}}\right)}\right\|_{F}=0 .
\end{aligned}
$$

It follows from Lemma 5 that there exists a $\gamma>0$ and a positive integer $M$ such that $\left\|e^{A\left(t_{i_{j+1}}-t_{0}\right)}\right\|_{F} \leq$ $\gamma\left(t_{i_{j+1}}-t_{i_{1}}\right)^{N-1} e^{a T j} \leq \gamma(j T)^{N-1} e^{a T j}, \forall j>M$, where $T$ is the upper bound for all the intervals $\left[t_{i_{j}}, t_{i_{j+1}}\right)$ and $a$ is the maximum real parts of the eigenvalues of $A$. Thus, according to (6) and (7), to prove (8), it suffices to prove

$$
\lim _{j \rightarrow \infty}\left[(j T)^{N-1}\right] e^{a T j}\left(\beta^{\frac{j-1}{N-1}-1}\right)=0 .
$$

Noticing that $0 \leq \beta<1$ and

$\left[(j T)^{N-1}\right] e^{a T j}\left(\beta^{\frac{j-1}{N-1}-1}\right)=T^{N-1} \beta^{\frac{-N}{N-1}} j^{N-1} e^{\left(\frac{\ln \beta}{N-1}+a T\right) j}$,

it follows directly from the fact that the exponential decay dominates the polynomial inflation that if $\frac{\ln \beta}{N-1}+a T<0$, i.e. $a<\frac{-\ln \beta}{(N-1) T}$, the equation in (9) holds.

Now we proceed to prove

$$
\lim _{j \rightarrow \infty} e^{A\left(t-t_{0}\right)} \otimes\left[\Phi\left(t, t_{0}\right)-\mathbf{1} v^{\mathrm{T}}\right]=0 .
$$

Let $i_{\ell+1}$ be the largest nonnegative integer satisfying $t_{i_{\ell+1}} \leq$ $t$, thus $t-t_{i_{\ell+1}} \leq T$ and $t_{i_{\ell+2}}>t$, the latter of which implies that $(\ell+1) T>t$, i.e. $\ell>\frac{t}{T}-1$. It follows from the fact that the transition matrix of system (4) is stochastic that

$$
\Phi\left(t, t_{0}\right)-\mathbf{1} v^{\mathrm{T}}=\Phi\left(t, t_{i_{\ell+1}}\right)\left(\Phi\left(t_{i_{\ell+1}}, t_{0}\right)-\mathbf{1} v^{\mathrm{T}}\right)
$$

and thus we have the inequality in (10) as shown on next page, where $\left\|\Phi\left(t, t_{i_{\ell+1}}\right) \otimes e^{A\left(t-t_{i_{\ell+1}}\right)}\right\|_{F}$ is upper bounded, say by $\eta$, this is because $t-t_{i_{\ell+1}} \leq T$ and all the nonzero elements in $L(t)$ are bounded; (11) is obtained from (10) by noting (6), (7), and Lemma 5, and (12) is obtained from (11) by noting $t_{i_{\ell+1}}-t_{0} \leq t-t_{0}$ and $\ell>\frac{t}{T}-1$. This combined with the fact that the exponential delay dominate the polynomial inflation that if $a<\frac{-\ln \beta}{(N-1) T}$, then

$$
\left(\Phi\left(t, t_{0}\right)-\mathbf{1} v^{\mathrm{T}}\right) \otimes e^{A\left(t-t_{0}\right)}
$$

approaches 0 at exponentially fast with the least rate of $\frac{\ln \beta}{(N-1) T}+a$, also

$$
\begin{aligned}
\lim _{t \rightarrow \infty} x(t) & =\lim _{t \rightarrow \infty}\left(\Phi\left(t, t_{0}\right) \otimes e^{A\left(t-t_{0}\right)}\right) x(0) \\
& =\left(\left(\mathbf{1} v^{\mathrm{T}}\right) \otimes e^{A\left(t-t_{0}\right)}\right) x(0) .
\end{aligned}
$$

That is, consensus for all the agents is achieved exponentially fast and the states of all the agents synchronize to $\left(v^{\mathrm{T}} \otimes e^{A\left(t-t_{0}\right)}\right) x(0)$.

Remark 1: Although consensus can be reached via information exchanges between neighboring agents, the trajectory for the agents may be oscillating or even divergent, which is dependent on the location of the eigenvalues of $A$.

Remark 2: Theorem 1 in fact gives a rough upper bound for the exponentially unstable of $A$, i.e. $a<$ 


$$
\begin{aligned}
& \left\|\left[\Phi\left(t, t_{0}\right)-\mathbf{1} v^{\mathrm{T}}\right] \otimes e^{A\left(t-t_{0}\right)}\right\|_{F} \\
= & \left\|\left[\Phi\left(t, t_{i_{\ell+1}}\right)\left(\Phi\left(t_{i_{\ell+1}}, t_{0}\right)-\mathbf{1} v^{\mathrm{T}}\right)\right] \otimes\left[e^{A\left(t-t_{i_{\ell+1}}\right)} e^{A\left(t_{i_{\ell+1}}-t_{0}\right)}\right]\right\|_{F} \\
= & \left\|\left[\Phi\left(t, t_{i_{\ell+1}}\right) \otimes e^{A\left(t-t_{i_{\ell+1}}\right)}\right]\left[\left(\Phi\left(t_{i_{\ell+1}}, t_{0}\right)-\mathbf{1} v^{\mathrm{T}}\right) \otimes e^{A\left(t_{i_{\ell+1}}-t_{0}\right)}\right]\right\|_{F} \\
\leq & \left\|\Phi\left(t, t_{i_{\ell+1}}\right) \otimes e^{A\left(t-t_{i_{\ell+1}}\right)}\right\|_{F} \cdot\left\|\left(\Phi\left(t_{i_{\ell+1}}, t_{0}\right)-\mathbf{1} v^{\mathrm{T}}\right) \otimes e^{A\left(t_{i_{\ell+1}}-t_{0}\right)}\right\|_{F} \\
\leq & \eta\left\|\Phi\left(t_{i_{\ell+1}}, t_{0}\right)-\mathbf{1} v^{\mathrm{T}}\right\|_{F} \cdot\left\|e^{A\left(t_{i_{\ell+1}}-t_{0}\right)}\right\|_{F}, \\
\leq & \mathbf{O}\left(\beta^{\frac{\ell}{N-1}} \cdot\left(t_{i_{\ell+1}}-t_{0}\right)^{N-1} \cdot e^{a\left(t_{i_{\ell+1}}-t_{0}\right)}\right) \\
\leq & \mathbf{O}\left(\beta^{\frac{t}{(N-1) T}} \cdot\left(t-t_{0}\right)^{N-1} \cdot e^{a t}\right) \\
= & \mathbf{O}\left(e^{\left(\frac{\ln \beta}{(N-1) T}+a\right) t} \cdot\left(t-t_{0}\right)^{N-1}\right)
\end{aligned}
$$

$\frac{-\ln \left(1-\mu^{\lfloor T / \tau\rfloor(N-1)}\right)}{(N-1) T}$. Although such a bound may not be tight, but it is independent of the switching mode. Apart from that, Theorem 1 extends the work concerning integrator agents corresponding to $A=0$ and $B=I_{n}$ in our work, see, e.g. Refs. [6], [16], to very general settings. Note that different from the asymptotical convergence results proved in those works, we prove the exponential consensus and can further specify the least convergence rate as well.

\section{Simulation Example}

We assume that there are a group of 5 agents and $n=$ 3 , i.e. each agents evolves in $\mathbb{R}^{3}$. Assume further that the interaction topology $G(t)$ switches every $1 s$ periodically, as shown in Figure 1, from $G a$ to $G b, G b$ to $G c$, and then $G c$ to $G a$. The union of $G a, G b$, and $G c$ has a spanning tree and thus the interaction topology is repeatedly jointly rooted. Moreover, to efficiently illustrate our result, we choose

$$
A=\left[\begin{array}{ccc}
a & 0 & -1 \\
0 & 0 & -2 \\
0 & 2 & 0
\end{array}\right] \text { and } B=\left[\begin{array}{llll}
1 & 0 & 0 & 1 \\
0 & 1 & 0 & 0 \\
0 & 0 & 1 & 0
\end{array}\right] \text {, }
$$

where $a$ is a number to be chosen. It can be easily computed that $\sigma(A)=\{a, 2 i,-2 i\}$, where $\sigma(A)$ denote the set of eigenvalues of $A$. In the simulation, the initial state of each agent is randomly chosen from interval $[-100,100] \times$ $[-100,100] \times[-100,100] \subset \mathbb{R}^{3}$.
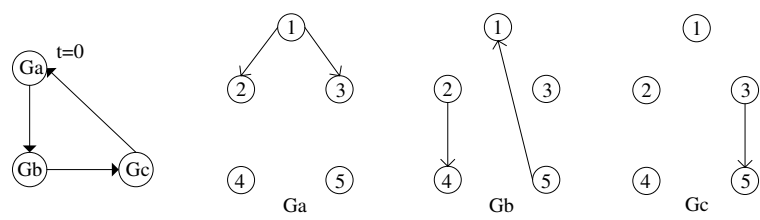

Fig. 1. Switching mode and three possible interaction topologies among 5 agents.

The following quantity is introduced to accurately investigate the process of consensus

$$
E(t)=\frac{1}{4} \sum_{i=2}^{5}\left|x_{1}-x_{i}\right| .
$$

Figure 2 shows that consensus for all the agents can be achieved if $a=0.1$ while it is shown by Figure 3 that consensus cannot be reached if $a=0.15$. This also shows that consensus can be reached even if $A$ has exponentially unstable mode (caused mainly by $a$ in this example), but such mode need to be sufficiently weak.

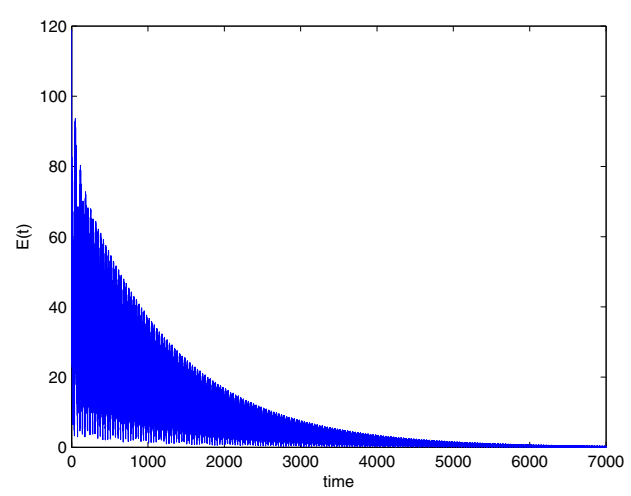

Fig. 2. Time evolution of $E(t)$ with $a=0.1$

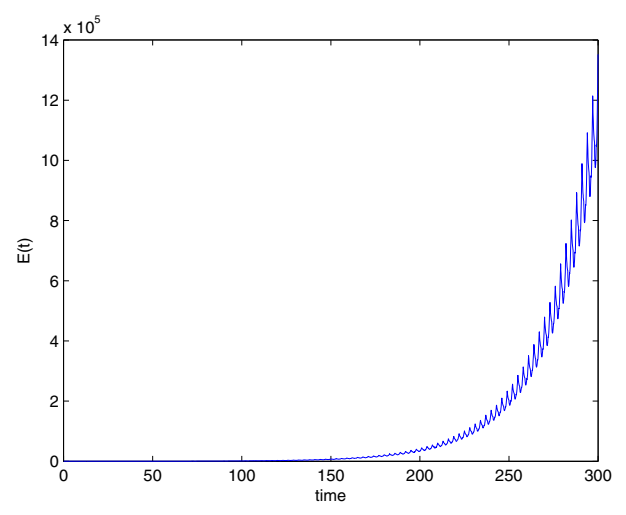

Fig. 3. Time evolution of $E(t)$ with $a=0.15$ 


\section{CONCLUSion}

We have studied in this paper the consensus control of generic linear multi-agent systems under directed dynamic topology. We have shown that via the full-state coupling among agents, consensus for all the agents can be achieved exponentially fast under a very general setting, i.e. the directed interaction topology is only required to be repeatedly jointly rooted and each individual system is allowed to have a sufficiently weak exponentially unstable mode. Further, we have specified a least convergence rate and a upper bound for such unstable mode, both of which are independent of the switching mode.

\section{REFERENCES}

[1] M. Cao, A. S. Morse, B. D. O. Anderson, "Agreeing asynchronously," IEEE Trans. Automat. Contr., vol. 53, no. 8, pp. 1826-1838, 2008.

[2] K. Cai and H. Ishii, "Quantized consensus and averaging on gossip digraphs," IEEE Transactions on Automatic Control, vol. 56, no. 9, pp. 2087-2100, 2011.

[3] S. Chatterjee and E. Seneta, "Towards consensus: some convergence theorems on repeated averaging," Journal of Applied Probability, vol. 14, pp. 89-97, 1977.

[4] Y. Gao and L. Wang, "Sampled-data based Consensus of continuoustime multi-agent systems with time-varying topology", IEEE Trans. Automat. Contr., vol. 56, no. 5, pp. 1226-1231, 2011.

[5] C. Godsil and G. Doyle, Algebraic Graph Theory. New York: Springer, 2001.

[6] A. Jadbabaie, J. Lin, and S. A. Morse, "Coordination of groups of mobile agents using nearest neighbor rules," IEEE Trans. Automat. Contr., vol. 48, no. 6, pp. 988-1001, 2003.

[7] T. Li and J. F. Zhang, "Consensus conditions of multi-agent systems with time-varying topologies and stochastic communication noises," IEEE Trans. Robot. Automat., vol. 55, no. 9, pp. 2043-2057, 2010.

[8] Z. Lin, B. Francis, and M. Maggiore, "State aggrement for continuoustime coupled nonlinear systems," SIAM J. Control Optim., vol. 46, no. 1, pp. 288-307, 2007.

[9] R. A. Horn and C. R. Johnson, Topics in Matrix Analysis, Cambridge, U.K.: Cambridge Univ. Press, 1991.

[10] L. Moreau, "Stability of multiagent systems with time-dependent communication links," IEEE Trans. Autom. Control, vol. 50, no. 2, pp. 169-182, 2005.
[11] W. Ni and D. Cheng, "Leader-following consensus of multi-agent systems under fixed and switching topologies," Systems Control Lett., vol.59, nos.3-4, pp. 209-217, 2010.

[12] R. Olfati-saber and R. M. Murray, "Consensus problems in networks of agents with switching topology and time-delays," IEEE Trans. Automat. Contr., vol. 49, no. 9, pp. 1520-1533, 2004.

[13] J. Qin, W. X. Zheng, and H. Gao, "Consensus of multiple second-order vehicles with a time-varying reference signal under directed topology", Automatica, vol. 47, no. 9, pp. 1983-1991, 2011.

[14] J. Qin, C. Yu, H. Gao, and X. Wang, "Leaderless consensus control of dynamical agents under directed interaction topology," in Proc. IEEE Conf. Decision Control and European Control Conf., Orlando, FL, 2011, pp. 1455-1460.

[15] J. Qin and H. Gao, "A sufficient condition for convergence of sampleddata consensus for double-integrator dynamics with nonuniform and time-varying communication delays," IEEE Trans. Automat. Contr., vol. 57, no. 9, pp. 2417-2422, 2012

[16] W. Ren and R. W. Beard, "Consensus seeking in multiagent systems under dynamically changing interaction topologies," IEEE Trans. Automat. Contr., vol. 50, no. 5, pp. 655-661, 2005.

[17] W. Ren and R. W. Beard, Distributed Consensus in Multi-vehicle Cooperative Control. London: Springer-Verilag, 2008.

[18] S. Tuna, "Synchronizing linear systems via partial-state coupling," Automatica, vol. 44, no. 8, pp. 2179-2184, 2008

[19] S. Tuna, "Conditions for synchronizability in arrays of coupled linear systems," IEEE Trans. Automat. Contr., vol. 54, no. 10, pp. 2416-2420, 2009.

[20] C. W. Wu, "Synchronization in networks of nonlinear dynamical systems coupled via a directed graph," Nonlinearity, vol. 18 , no. 3 , pp. 1057-1064, 2005.

[21] C. W. Wu, "Synchronization and convergence of linear dynamics in random directed newtorks," IEEE Trans. Automat. Contr., vol. 51, no. 7, pp. 1207-1210, 2006

[22] J. Wang, D. Chen, and X. Hu, "Consensus of multi-agent linear dynamical systems," Asian J. Control, vol. 10, no. 2, pp. 144-155, 2008.

[23] J. Wolfowitz, "Products of indecomposable, aperiodic, stochastic matrices," Proc. Amer. Math. Soc., vol. 14, pp. 733-737, 1963.

[24] K. You and L. Xie, "Network topology and communication data rate for consensusability of discrete-time multi-agent systems," IEEE Trans. Automat. Contr., vol. 56, no. 10, pp. 2262-2275, 2011.

[25] W. Yu, G. Chen, and M. Cao, "Some necessary and sufficient conditions for second-order consensus in multi-agent dynamical systems," Automatica, vol. 46, no. 6, pp. 1089-1095, 2010. 